\title{
Simulations of the L-H transition on experimental advanced superconducting Tokamak
}

\author{
Jan Weiland \\ Department Applied Physics, Chalmers University of Technology and Euratom-VR Association, \\ S41296 Gothenburg, Sweden
}

(Received 11 September 2014; accepted 3 November 2014; published online 3 December 2014)

We have simulated the L-H transition on the EAST tokamak [Baonian Wan, EAST and HT-7 Teams, and International Collaborators, "Recent experiments in the EAST and HT-7 superconducting tokamaks," Nucl. Fusion 49, 104011 (2009)] using a predictive transport code where ion and electron temperatures, electron density, and poloidal and toroidal momenta are simulated self consistently. This is, as far as we know, the first theory based simulation of an L-H transition including the whole radius and not making any assumptions about where the barrier should be formed. Another remarkable feature is that we get $\mathrm{H}$-mode gradients in agreement with the $\alpha-\alpha_{\mathrm{d}}$ diagram of Rogers et al. [Phys. Rev. Lett. 81, 4396 (1998)]. Then, the feedback loop emerging from the simulations means that the L-H power threshold increases with the temperature at the separatrix. This is a main feature of the C-mod experiments [Hubbard et al., Phys. Plasmas 14, 056109 (2007)]. This is also why the power threshold depends on the direction of the grad B drift in the scrape off layer and also why the power threshold increases with the magnetic field. A further significant general H-mode feature is that the density is much flatter in H-mode than in L-mode. (C) 2014 AIP Publishing LLC. [http://dx.doi.org/10.1063/1.4901597]

The understanding of the L-H transition in tokamaks is still one of the outstanding issues in fusion transport research. We have here used data from the EAST tokamak ${ }^{1-3}$ which we have recently studied with our transport code ${ }^{4}$ to improve our general understanding of the $\mathrm{L}-\mathrm{H}$ transition. ${ }^{5-12}$ A main reason is that the performance of the projected ITER depends strongly on the height of the edge temperature pedestal. ${ }^{13}$ In the present design, a pedestal temperature of about $4 \mathrm{keV}$ is needed. The achievement of this temperature may be critical, i.e., some but not all theories predict such a high temperature. Thus, we need to understand both the H-mode power threshold and the height of the pedestal. A lot of theory work has been devoted to this problem. ${ }^{14-21} \mathrm{~A}$ useful review of both experimental background and theoretical models was given in Ref. 14. The most ambitious models have been derived through nonlinear simulations of edge turbulence. ${ }^{16,17}$ In particular, the dimensionless parameters $\alpha=-\mathrm{q}^{2} \operatorname{Rd} \beta / \mathrm{dr}$, where $\beta$ is the usual plasma beta (ideal MHD parameter) and $\alpha_{\mathrm{d}}=\mathrm{v}_{\mathrm{d}} \mathrm{t}_{\mathrm{ib}} / \mathrm{L}$, where $\mathrm{v}_{\mathrm{d}}$ is the ion diamagnetic velocity, $t_{i b}$ is the ideal toroidal ITG growth time, and $\mathrm{L}$ is a characteristic turbulence scale length prop to $\mathrm{q}\left(\mathrm{R} \rho_{\mathrm{s}} \nu_{\mathrm{ei}} / \Omega_{\mathrm{ce}}\right)^{0.5}$ were successfully used to characterize the edge. ${ }^{17}$ In an $\alpha-\alpha_{d}$ plane, regions of $\mathrm{H}$-mode as well as density limit instability and ideal MHD instability could be identified. ${ }^{17}$ Their H-mode region is actually in fairly good agreement with the $\mathrm{H}$-mode in $\mathrm{C}$-mod as seen in Ref. 10 . Actually, several measurement points in C-mode were just below the H-mode regime in Ref. 17 so clearly we can also accept results just below this region as H-modes in our simulations. In recent more detailed studies of the pedestal, it has been found that both kinetic ballooning modes ${ }^{22,23}$ and Peeling modes are active. ${ }^{20,21}$

We use the advanced reactive toroidal drift wave model described in Ref. 24. For the kinetic ballooning modes, we use the fluid formulation in Ref. 23. For the toroidal momentum transport, we use the recently derived toroidal symmetry breaking effects. ${ }^{25-27}$ The basic features are

Saturation level without flow shear (Ref. 24)

$$
\frac{e \phi}{T_{e}}=\frac{\gamma}{k_{\theta} c_{s} k_{r} \rho}=\frac{\gamma}{\omega_{* e}} \frac{1}{k_{r} L_{n}} .
$$

With the Waltz rule, ${ }^{28,29}$ subtracting the flow shear rate, we have

$$
\gamma \rightarrow \gamma-\omega_{E x B}
$$

and

$$
\frac{e \phi}{T_{e}}=\frac{\gamma-\omega_{E x B}}{k_{\theta} c_{s} k_{r} \rho}=\frac{\gamma-\omega_{E x B}}{\omega_{* e}} \frac{1}{k_{r} L_{n}} .
$$

We here consider real space so we take $k_{\mathrm{r}} \approx 1 / \mathrm{L}_{\mathrm{r}}$, where $\mathrm{L}_{\mathrm{r}}$ is the radial correlation length and analogously for the poloidal lengthscale. This leads for the transport from the simplest electrostatic ITG mode to ${ }^{24}$

$$
\chi_{i}=\frac{1}{\eta_{i}}\left(\eta_{i}-\frac{2}{3}-\frac{10}{9 \tau} \varepsilon_{n}\right) \frac{\left(\gamma-\omega_{E x B}\right)^{3} / k_{r}^{2}}{\left(\omega_{r}-\frac{5}{3} \omega_{D i}\right)^{2}+\left(\gamma-\omega_{E x B}\right)^{2}} .
$$

We write down Eq. (3) just in order to illustrate how the total saturation level, due to the combined influence of radial $\mathrm{E} \times \mathrm{B}$ convection and the Waltz rule enter diffusivities. Of course the full model contains much more physics but these are the crucial points in the comparison with a turbulence code $^{17}$ which treats the complete saturation problem for many modes on the turbulence time scale. Here, we use only one mode at the inverse correlation length. Actually, the form of (1) implies this since it has been obtained by 
balancing the growth rate with the convective $\mathrm{E} \times \mathrm{B}$ nonlinearity which is assumed to be entirely stabilizing. Thus, we have to be at the source of the turbulence where no energy comes in from more unstable modes and no energy is reflected from the boundaries in k-space. This was confirmed by extensive $2 \mathrm{~d}$ turbulence simulations in the end of the 1980s (see Ref. 24, and references therein). Since we can obtain the simple mixing length transport from the diagonal part of (3) by going in the limit of large growth rate and ignoring flow shear we also conclude that the same conditions are valid for that. The flow shear rate is given by ${ }^{30}$

$$
\omega_{E x B}=\frac{r}{q} \frac{\partial}{\partial r}\left(\frac{q V_{E \theta}}{r}\right) .
$$

In order to calculate the flow shear rate as $\mathrm{V}_{\mathrm{E} \theta}=\mathrm{E}_{\mathrm{r}} / \mathrm{B}$, we need to know the radial electric field obtained from radial force balance

$$
E_{r}=B_{\theta} V_{\phi}-B_{\phi} V_{\theta}+\frac{1}{e Z_{i n}} \frac{\partial P_{i}}{\partial r} .
$$

Here, we need to know the poloidal and toroidal plasma flows so we include transport equations for them. The toroidal flow is described by

$$
\begin{aligned}
m_{i} N_{i} & \left(\frac{\partial}{\partial t}+2 \mathbf{V}_{D n} \cdot \vec{\nabla}\right) \delta \mathrm{v}_{\|} \\
= & -m_{n} N_{i} \overrightarrow{\mathbf{u}}_{E} \cdot \nabla V_{\| 0}-\left[\hat{e}_{\|} \cdot \nabla+e V_{\| 0} \frac{m_{i} \mathbf{V}_{D_{i}}}{T_{i}} \cdot \nabla\right] \\
& \times\left(\delta p_{i}+e N_{i} \phi-\frac{\omega+\omega_{* e}\left(1+\eta_{e}\right) / \tau}{k_{\|} c} A_{\|}\right)
\end{aligned}
$$

or

$$
\begin{aligned}
\delta \mathbf{v}_{\|}= & -\frac{k_{\theta} D_{B}}{\omega-2 \omega_{D i}} \frac{d \mathbf{V}_{\| 0}}{d r}+\frac{\left\langle k_{\|}\right\rangle+\omega_{D i} V_{\| 0} /\left(\tau \cdot c_{s}^{2}\right)}{\omega-2 \omega_{D i}} \\
& \times\left(\delta p+n e \phi-\frac{\omega+\omega_{* e}\left(1+\eta_{e}\right) / \tau}{k_{\|} c} A_{\|}\right) /\left(m_{i} n_{i}\right),
\end{aligned}
$$

where

now

$$
\mathbf{V}_{D i}=2 \frac{T_{i}}{e B^{2}} \times \nabla B ; \quad D_{B}=\frac{T_{e}}{e B}
$$

$$
\Gamma_{\|}=\left\langle\mathrm{v}_{\mathrm{Er}} \delta \mathrm{v}_{\|}\right\rangle \approx \Gamma_{\phi}
$$

Here, the convective magnetic drift term in the left hand side can either be obtained from a gyrofluid approach ${ }^{31}$ or from fluid equations including the stress tensor. ${ }^{26,27}$ It was recently found that electromagnetic effects increase the toroidal momentum pinch. ${ }^{32}$ We then calculate the transport of toroidal momentum in the usual way

$$
\frac{\partial V_{\phi}}{\partial t}+\frac{\partial}{\partial r} \Gamma_{\phi}=S_{v}
$$

where the source term $\mathrm{S}$ is zero for EAST in the absence of neutral beam heating.
The poloidal rotation is calculated in a similar way.

The poloidal flux is given by the Reynolds stress as ${ }^{24}$

$$
\Gamma_{\theta}=\left\langle\mathrm{v}_{\operatorname{Er}} \mathrm{v}_{\theta}\right\rangle=-D_{B}^{2} k_{r} k_{\theta} \frac{1}{2} \widehat{\phi}^{*}\left[\widehat{\phi}+\frac{1}{\tau} \widehat{P}_{i}\right]+c . c
$$

and thus

$$
\begin{gathered}
\frac{\partial V_{\theta}}{\partial t}+\frac{\partial}{\partial r} \Gamma_{\theta}=S_{v}, \\
S_{v}=0.67 \nu_{i i}\left(\mathrm{v}_{\theta}-\mathrm{v}_{\mathrm{nc}}\right) / \varepsilon, \quad \varepsilon=r / R .
\end{gathered}
$$

We here use the neoclassical rotation, $\mathrm{v}_{\mathrm{nc}}$, according to Ref. 30.

Thus, the sink term in our case only consists of the neoclassical damping. Here, also the diamagnetic drift was included as a convected velocity. A very important point is here that the temperature perturbation enters dynamically in (7a). This will give us a feedback loop, since a temperature gradient gives a temperature perturbation which generates a shear flow. The shear flow reduces the instability and thus the temperature flux is reduced, giving an increased temperature gradient, etc.,

$$
\begin{gathered}
\Gamma_{T}=-\chi \frac{d T}{d r}=\chi \frac{T}{L_{t}}, \\
L_{T}=-\frac{T}{d T / d r} .
\end{gathered}
$$

We remember that the flux here is given by the interior heating. Thus, good confinement is accompanied by steep gradient in (8a). Furthermore, the pressure perturbation in (7a) contains the temperature perturbation $\delta \mathrm{T}$ where the convective part is given by

$$
\delta T_{c}=-\xi \frac{d T}{d r}=-\eta \frac{\omega_{* n}}{\omega} q \phi,
$$

where $\xi$ is the $\mathrm{E} \times \mathrm{B}$ displacement and $\eta=\mathrm{L}_{\mathrm{n}} / \mathrm{L}_{\mathrm{t}}$. Subindex $\mathrm{n}$ indicated that the diamagnetic drift is taken with only the density gradient. We now observe from (8b) that for given thermal conductivity and fixed heat source, $\mathrm{L}_{\mathrm{T}}$ depends mainly on T. Now, the L-H transition takes place just inside the separatrix. There the outer temperature boundary is given by the temperature at the separatrix. Thus, a lower separatrix temperature boundary leads to a shorter $\mathrm{L}_{\mathrm{T}}$ and a larger $\eta$. Thus, the temperature perturbation in (8), entering in the pressure perturbation in $(7 \mathrm{a})$, is reduced for higher temperature at the separatrix and vice versa.

A reduced temperature at the separatrix would give an increased temperature perturbation in (8) and this will actually lead to a positive feedback, i.e., an increased Reynolds stress will give increased poloidal rotation and accordingly shear flow. This will reduce the thermal conductivity in (8a) and, since the flux is fixed, this will reduce $\mathrm{L}_{\mathrm{T}}$. Now for this feedback to work, the temperature perturbation must become the dominant part of (7a). This is what determines the power threshold since an increased heating gives a larger flux corresponding to a shorter $\mathrm{L}_{\mathrm{T}}$ in (8a). Thus, our picture is clear. We have a situation where the power 
threshold increases with the edge temperature. This is also consistent with the dependence of the power threshold on the direction of the grad B drift since that changes the edge temperature as shown in Ref. 11. Since the temperature at the separatrix increases with the magnetic field, $\mathrm{B}$, this also explains why the power threshold increases with $B$. All these aspects are in agreement with Ref. 11.

In our scalings of power threshold with $\mathrm{B}$, we have implemented the empirical scaling $\mathrm{T}_{\text {sep }} \sim \mathrm{B}$ in the code. It is also important to recognize the role of the fixed temperature at the separatrix as the reason why $\mathrm{L}_{\mathrm{T}}$ decreases when we increase the heating. The poloidal momemtum flux (7a) is also relevant for internal transport barriers ${ }^{24}$ and again the temperature gradient length scale is the key parameter. However, in the core, both the temperature and its gradient can increase at the same time when we increase the heating, leaving the effect on $\mathrm{L}_{T}$ open. This means that we need something more, like, e.g., small magnetic shear, to reduce $\mathrm{L}_{\mathrm{T}}$.
In our full model, we include slab and toroidal ITG, trapped electron modes driven by charge separation, compression or collisions, resistive ballooning modes, ${ }^{18,24}$ MHD and kinetic ballooning modes, and peeling modes. All these modes are discussed in Ref. 24. The relation between drift type and MHD type modes is illustrated by the parallel electric field which follows from the relation between electrostatic and magnetic potentials. To obtain this, we use the free electron continuity and parallel momentum equations

$$
\begin{gathered}
\frac{\partial n_{e f}}{\partial t}+\nabla \cdot\left[n_{e f}\left(\mathbf{v}_{\mathbf{E}}+\mathbf{v}_{* \mathbf{e}}+\mathbf{v}_{\| 0} \frac{\delta \mathbf{B}_{\perp}}{\mathrm{B}}+\mathbf{v}_{\|} \widehat{\mathbf{e}}_{\|}\right)\right]=0, \\
\frac{\partial n_{e f}}{\partial t}=\frac{e}{T_{e}}\left(\phi+\frac{\omega_{* e}-\omega}{k_{\|}} A_{\|}\right)+i \frac{\mathbf{v}_{\| \mathrm{e}}}{\mathrm{k}_{\|} D_{e}},
\end{gathered}
$$

which are combined to give

$$
\frac{e A_{\|}}{T_{e}}=k_{\|} \frac{\omega-\omega_{* e}}{\omega\left(\omega-\omega_{* e}\right)+\omega_{D e}\left(\omega_{* e T}-\omega\right)-\frac{k_{\|} k_{\theta} T_{e}}{e^{2} B n_{0}} \frac{\partial \mathrm{J}_{\| 0}}{\partial \mathrm{r}}-\mathrm{k}_{\perp}^{2} \rho_{s}^{2} k_{\|\|_{\mathrm{A}}^{2}}^{2}(1-i \delta)} \frac{e \phi}{T_{e}},
$$

$$
\delta=\frac{\omega-\omega_{D e}}{k_{\|}^{2} D_{e}} \quad D_{e}=\frac{T_{e}}{m_{e} \nu_{e}} .
$$

Here, Eq. (10) connects the electrostatic and electromagnetic potentials and thereby gives the parallel electric field. The magnetic drift in the denominator leads to quadratic magnetic drifts in the final eigenvalue equation. Accordingly, Eq. (10) produces the kinetic ballooning mode in a fluid description. ${ }^{23}$ Equation (10) also includes the kink driving current gradient term $\mathrm{dJ}_{\| 0} / \mathrm{dr}$. (The purpose of this is not to include the global kink modes of MHD type but rather the peeling mode which has modenumbers similar to the MHD ballooning mode.) Because of this we can, to lowest order, use the usual ballooning formalism. ${ }^{14,15}$ Thus, we use the descriptions in Refs. 33-35 for solving the linear eigenvalue problem. We use a parameter dependent correlation length (Ref. 34) which gives typical drift wave correlation lengths in the core but more MHD like correlation lengths in the edge. We also include the dependence of the correlation length on flow shear as derived in Ref. 35. A special correlation length for electron modes was also given in Ref. 36. It includes dependence on collisions which are important for the resistive ballooning modes. ${ }^{18,24,37}$

The collisions on free electrons in Eq. (10) includes resistive ballooning modes in our description. Thus, the total model includes ITG modes (slab and toroidal), trapped electron modes (driven by charge separation (ubiquitous) and compression, collisionless or collisional) are included. We also include electromagnetic effects on all these modes. Electromagnetic effects introduce also kinetic ballooning modes and as discussed above Peeling modes. The model includes also ideal MHD ballooning modes. The full model is used everywhere so which modes dominate depends on the situation. Thus, ITG modes tend to dominate in the core while resistive ballooning modes tend to dominate in the edge except, of course, on the edge barrier where kinetic ballooning and Peeling modes dominate.

The poloidal and toroidal momenta are both simulated and are then combined to give the radial electric field ${ }^{24}$ as discussed above. This formulation has also been successful in simulating internal transport barriers on $\mathrm{JET}^{38}$ as discussed in Refs. 24 and 36.

We have simulated the EAST shot 38300 including self consistent variations of $\mathrm{T}_{\mathrm{i}}, \mathrm{T}_{\mathrm{e}}, \mathrm{n}_{\mathrm{e}}, \mathrm{V}_{\theta}$, and $\mathrm{V}_{\varphi}$. Nominal parameters are $\mathrm{B}_{\mathrm{T}}=2.77 \mathrm{~T}$ and heating power totally 0.77 MW with 0.19 MW on electrons (Fig. 1).

We first show the standard case. As pointed out above, the power threshold increases with the edge temperatures. The error bars in the measurements are substantial so the boundary temperatures in our standard case have been chosen as $30 \%$ of the nominal values.

In Fig. 1, the dotted lines represent initial profiles of Lmode type, chosen so low that the L-H transition becomes evident and without any initial trace of a barrier. The density, Fig. 1(c), is clearly much flatter than an L-mode profile would be. The L-H transition is triggered by the poloidal spinup, as discussed above. We note that the poloidal spinup is very localized to regions of steep barriers. The reason is that the neoclassical damping dominates in other regions. The toroidal rotation, Fig. 1(d), on the other hand, is much wider. It actually has a stronger effect on the width of the transport barrier than the poloidal rotation. However, in some cases, it does not develop at all. In these simulations, the initial profile of poloidal rotation has been entirely 

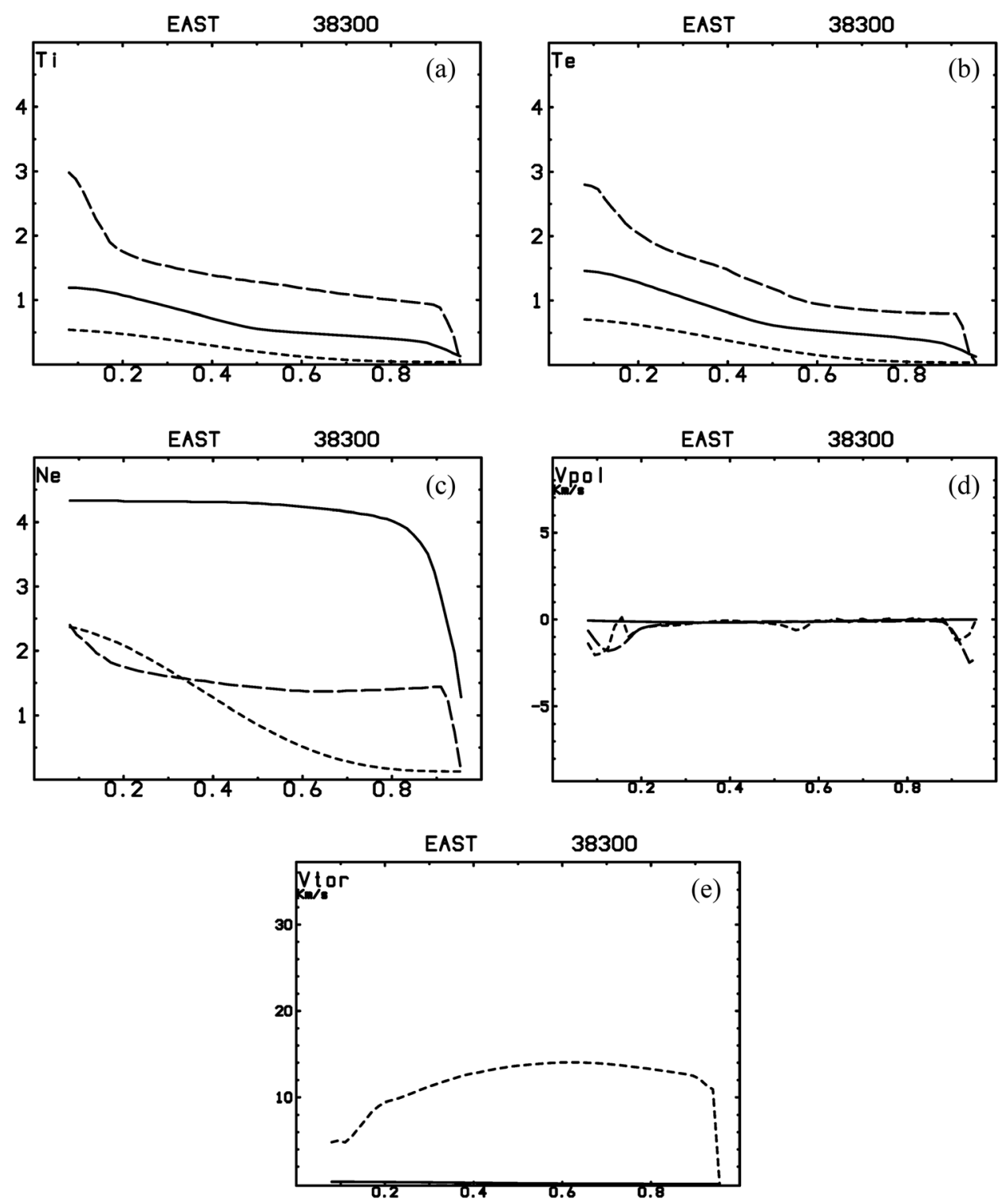

FIG. 1. (a) Simulated Ti, dashed line with initial profile, dotted line and experimental Ti full line. (b) Simulated $\mathrm{T}_{\mathrm{e}}$, the line patterns are the same as in (a). (c) Simulated $n_{e}$ corresponding to Fig. 2(a). (d) Simulated poloidal rotation (dotted) and final neoclassical profile (dashed). (e) Simulated toroidal rotation (dotted) initial profile (full).

neoclassical while the toroidal rotation had a boundary (fixed) due to intrinsic rotation. The choice of this boundary condition has been guided by Refs. 39 and 40. From Ref. 40, we find the estimate

$$
U_{t}=\rho_{*}\left(R / L_{T}\right)^{2}
$$

where $\mathrm{U}_{\mathrm{t}}=\mathrm{V}_{\mathrm{t}} / \mathrm{c}_{\mathrm{s}}$ and $\mathrm{R} / \mathrm{L}_{\mathrm{t}} \sim 30$ in the edge region. With $\rho_{-\mathrm{x}}$ $\sim 10^{-3}$, this gives the estimate $\mathrm{U}_{\mathrm{t}} \sim 10^{-1}$. However, this estimate is rather uncertain. Actually, an $U_{t} \sim 10^{-4}$ is typically sufficient for a strong excitation of toroidal rotation and, due to the toroidal momentum pinch, a higher boundary does not make much difference. However, the projection of the neoclassical rotation in the toroidal direction is usually about $10^{-6}$ and such a boundary rotation is not enough for having strong toroidal rotation. The interior rotation is then built up by the toroidal momentum pinch which is enhanced by electromagnetic effects. Then, the sign of the toroidal rotation is important at the trigger of the barrier, since it can reduce or enhance the radial electric field due to the poloidal rotation. We also notice from
Fig. 2(d) that there is a poloidal spinup also near the axis. It here produces an internal barrier in the ion temperature. This is not present in the timeslice we have used here but EAST 38300 has sometimes been characterized as an internal barrier shot so the data are probably close to the triggering of an internal barrier.

Increasing the total B improves the confinement which allows steeper gradients. At the same time, also the temperatures at the separatrix increase and the width of the pedestal increases.

From data in Ref. 11, we conclude that $\mathrm{T}_{\text {sep }} \sim$ B. Fig. 2 shows a simulation where the total $\mathrm{B}$ field was increased by $50 \%$.

Since our standard case with nominal heating (0.77 MW) is about $20 \%$ above threshold and the simulations with $50 \%$ higher $\mathrm{B}$ is at the threshold with $30 \%$ increased power we conclude that the power threshold has increased by about $50 \%$. Thus, we have an approximately linear scaling of power threshold with total $\mathrm{B}$. We note that the temperature pedestal is about a factor of 2 higher in this case. We have also looked at the $\mathrm{q}$ scaling. However, the results are fairly insensitive. In 

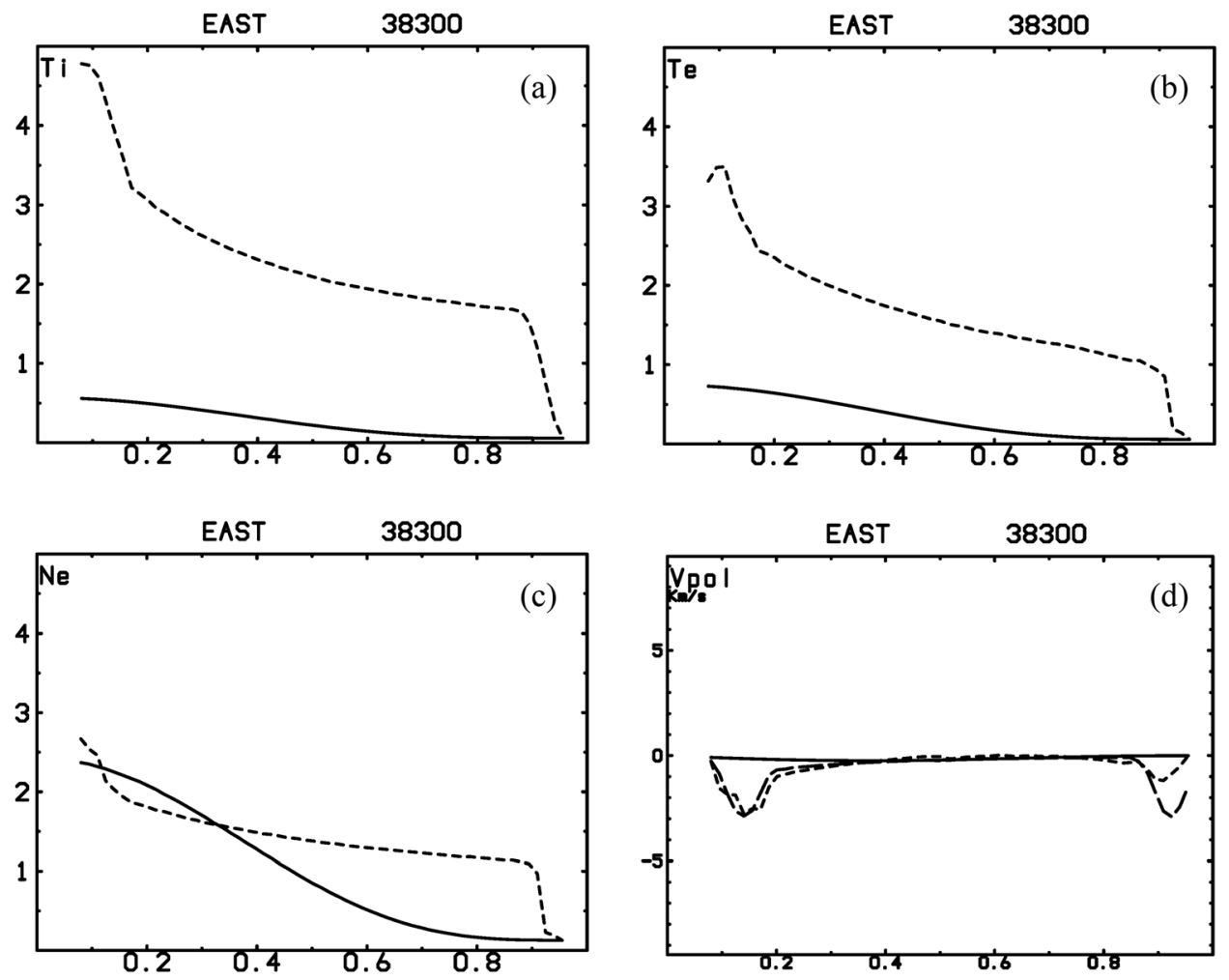

FIG. 2. (a) Ion temperature for $\mathrm{B}_{\mathrm{t}}=4.1 \mathrm{~T}, \mathrm{P}=1 \mathrm{Mw}$. Initial profilefull line, final profile-dotted. (b) Electron temperature corresponding to (a). (c) Electron density corresponding to (a). (d) Poloidal rotation corresponding to (a). (e) Toroidal rotation corresponding to (a).

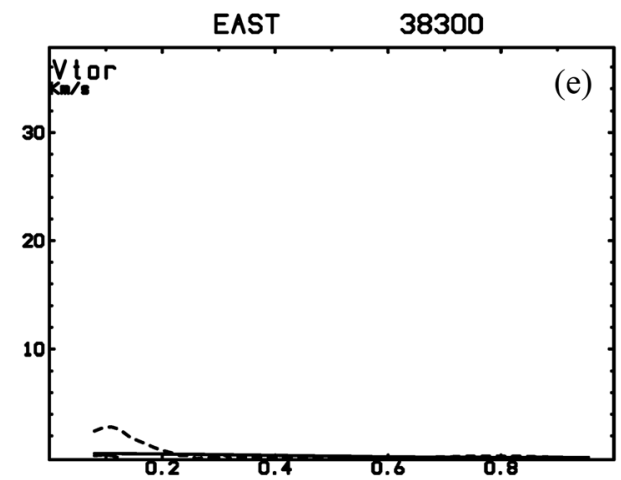

any case, the toroidal rotation remains if we reduce $\mathrm{q}$ by $25 \%$ and the density pedestal increases somewhat.

We note that in this case the density pedestal increases with current, i.e., when $\mathrm{q}$ is reduced. We also have an excitation of the toroidal rotation in this case although it is not as strong as in some other cases.

The scalings deduced from these data for pedestal width $\delta$ and height are

and

$$
T_{\text {ped }} \propto B^{2}
$$

While the case shown for 50\% increase of B (Fig. 3) is close to the actual threshold (1 MW) the standard case is about $20 \%$ above threshold. As pointed out above, the
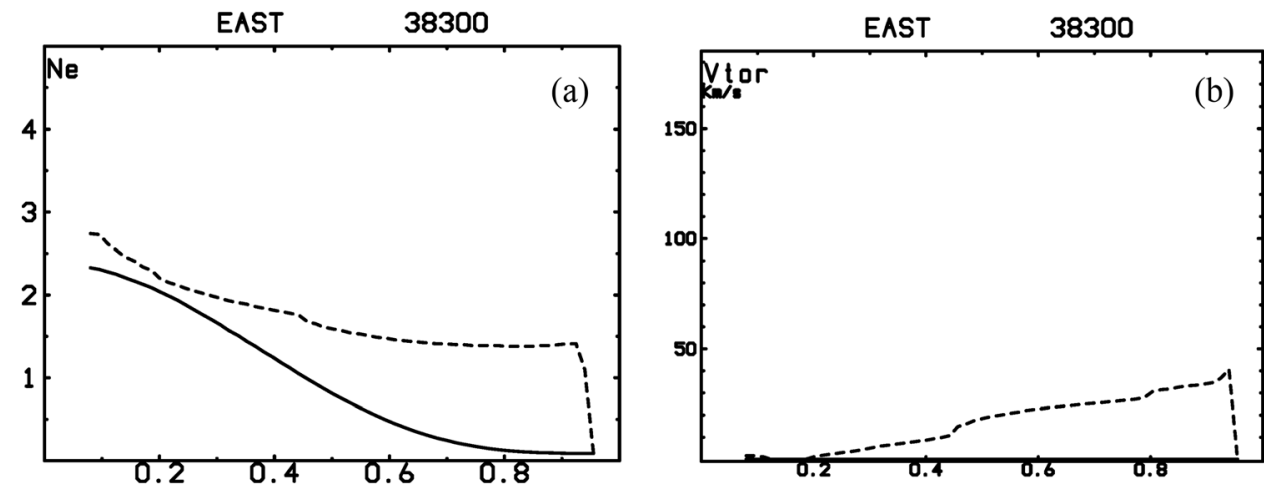

FIG. 3. (a) Electron density corresponding to Fig. 2(c) but for $25 \%$ reduced q. (b) Toroidal rotation corresponding to Fig. 3(a). 
temperature at the separatrix was in Ref. 11 found to scale as

$$
T_{\text {sep }} \propto B
$$

Using this scaling, our simulations give the scaling

$$
P_{\text {thres }} \propto B
$$

which is also in agreement with Ref. 11.

As mentioned in the Introduction, we have entered our pedestal data in H-mode into the diagram of Rogers et al. ${ }^{17}$ The result is shown in Fig. 4. Although the effective collision frequency of trapped electrons normalized by the magnetic drift frequency at the edge is typically just below 100 in these plasmas, it turns out that $\nu_{*}=\nu_{\mathrm{ei}} / \omega_{* \mathrm{e}}$ starts from being of order 30 initially (L-mode) but finally becomes of order $10^{-2}$ in $\mathrm{H}$ mode because of steep density gradient. We note also that an increased line average density will increase the threshold power due to the reduction of heating power per particle. This is, however, a bulk effect. What we consider here is the temperature flux which actually is reduced by an increased density. Thus, we are here discussing only temperature flux dynamics and the dependence on density would enter but has to be added to yield the correct temperature source.

Fig. 4 shows the modified $\alpha_{d}-\alpha$ diagram of Rogers et al. ${ }^{17}$ where we have entered points from the H-mode barrier in our simulations. It is actually expected that only a limited region of the barrier should occupy the H-mode region in Fig. 4. This is enough since a region of very good confinement will plug the profile and give a barrier. The upper area corresponds to MHD instability. The two points from "enhanced gaspuff" are from the same simulation at neighbouring gridpoints. This further confirms that the resolution

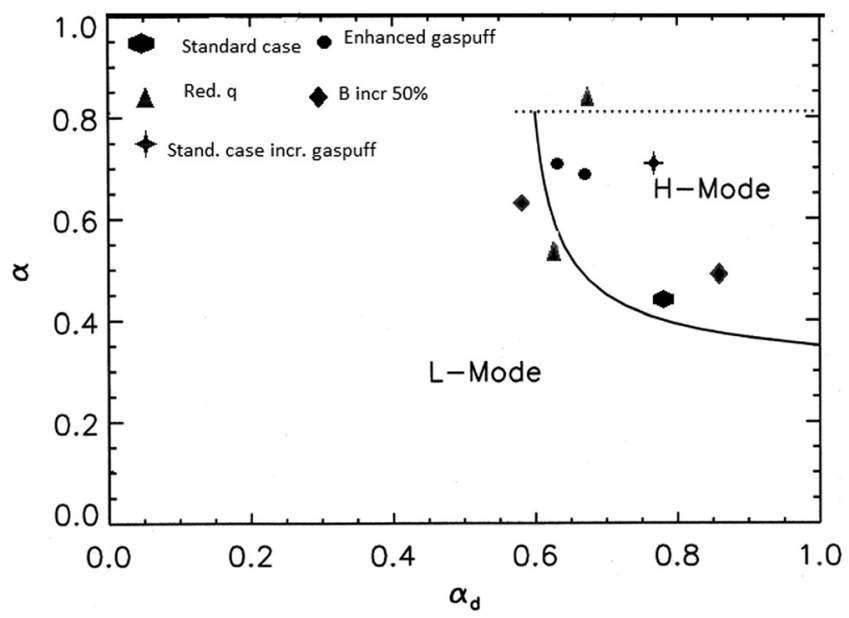

FIG. 4. The modified $\alpha_{\mathrm{d}}-\alpha$ diagram of Rogers et al. ${ }^{17}$ where we have entered points from the H-mode barrier in our simulations. It is actually expected that only a limited region of the barrier should occupy the H-mode region in Fig. 5. This is enough since a region of very good confinement will plug the profile and give a barrier. The upper area corresponds to MHD instability. The two points from "enhanced gaspuff" are from the same simulation at neighbouring gridpoints. This further confirms that the resolution is sufficient. We note that we have recovered the $\mathrm{H}$-mode regime obtained by local turbulence simulations in a global transport code. Adapted with permission from Rogers et al., Phys. Rev. Lett. 81, 4396 (1998). Copyright 2014 by the American Physical Society. is sufficient. We note that we have recovered the $\mathrm{H}$-mode regime obtained by local turbulence simulations in a global transport code.

We have here not included simulations ending up with $\alpha_{\mathrm{d}}$ larger than 1 although such cases have occurred. The reason is that in Ref. 17 a nonlinear instability, not included here, was found in this region. So far we have ignored ETG modes. ${ }^{41,42}$ This has been motivated by the fact that ETG modes were not included in Ref. 17 which is our main point of comparison. We are not aware of any study of the L-H transition where the ETG mode has been considered. However, in a recent study of EAST $38300^{4}$ ETG modes were found to be important. Thus, we have added a study of the effect of the ETG mode on the L-H transition. As it turned out, the power threshold was increased by about $20 \%$ when the ETG mode was included. Moreover, we had to increase the edge boundary of the toroidal rotation somewhat. However, it was still well below the expectations from Refs. 39 and 40 so the general picture still holds. We have also compared the threshold at increased $\mathrm{B}$. There the values reached in the $\alpha_{d}-\alpha_{h}$ diagram is about $15 \%$ below the $H$ mode boundary in Fig. 5. However, according to Ref. 10, this was also the case for $\mathrm{C}$-mod $\mathrm{H}$-modes. This makes the value of the increase of the threshold with $\mathrm{B}$ somewhat more uncertain. Another point is that our model for ETG modes has been taken from Ref. 42 which is based on numerical results from Ref. 41. These results have not been tested according to our general procedure of finding the fastest growing mode normalized by the drift frequency as discussed above (after Eq. (3)). Thus, this part of the investigation is somewhat more uncertain although the results appear to be in the line with the previous.

Although we make extensive comparison with experimental scalings, our main result is actually that we have shown that it is possible to use a global transport code to get agreement with local results from a fully nonlinear turbulence code. An important point is that this agreement is completely spontaneous in self consistent simulations where the same model and grid size are used everywhere, i.e., we do not put in any a priori knowledge about which results are expected. We have here also resolved several remaining

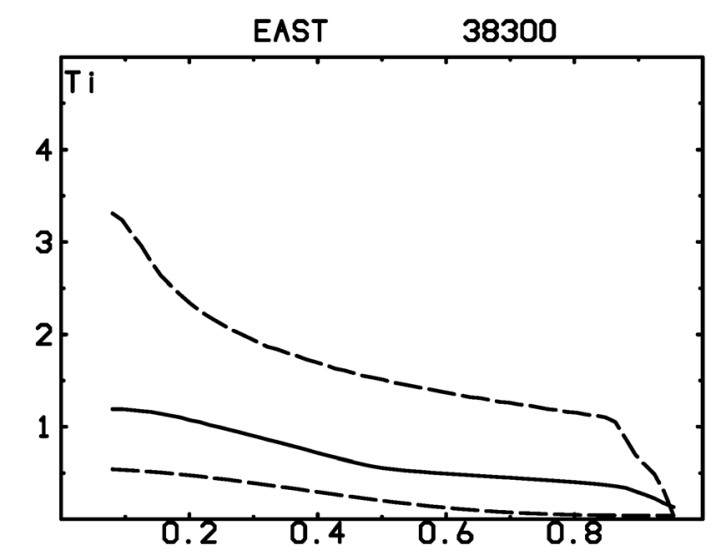

FIG. 5. Ion temperature profile including effects of ETG modes at experimental B. The heating power is here the experimental which is exactly at the power threshold (symbols as in Fig. 2). 
questions regarding the $\mathrm{L}-\mathrm{H}$ transition and $\mathrm{H}$-mode pedestal. The most important aspect is that the temperature at the separatrix has been identified as the key parameter for the L-H transition. A low separatrix temperature gives a low power threshold. This has come out of the simulations and is also seen analytically in the feedback loop for the transition. This is also consistent with the dependence of the L-H transition on the direction of the grad B drift. It also leads to a power threshold that increases with magnetic field in agreement with experiments. ${ }^{11}$ It is natural that the temperature at the separatrix increases with $\mathrm{B}$, since the magnetic field is used to confine the plasma. This is true both for Bohm and Gyro Bohm scaling. However, it seems that here also processes in the scrape off layer, including the direction of the grad B drift are important since these influence the temperature at the pedestal. Thus, our results on the consistent dependence of the threshold on the direction of the grad B drift follow from the experimental result that the temperature at the separatrix increases for reversed direction of the magnetic field. Thus, our result is that it is the temperature at the separatrix which is the key parameter for the L-H transition. We have here shown how a fluid model containing both poloidal and toroidal momentum transport can describe the formation of the edge transport barrier in a self-consistent simulation of five channels, ion and electron temperature, electron density, and poloidal and toroidal momenta. The L-H transition is triggered by the poloidal spinup which has previously been found to give both internal and edge transport barriers on JET. ${ }^{36}$ The reason why the toroidal momentum is insignificant in Fig. 2(e) is not clear. The edge boundary is almost the same as in Fig. 1(e). However, in the reference simulation (Fig. 1(e)), the poloidal and toroidal drives are typically comparable, while the toroidal drive is insignificant over the whole profile in Fig. 2(e). Electron modes dominate transport in the whole transport barrier region. Our correlation length for electron modes also increases (more global modes) near the edge which is suitable for describing the MHD type modes on the H-mode barrier. Electromagnetic effects have recently been found to be important for the toroidal momentum pinch $^{32}$ which can also be an important part of the dynamics. The model for elongation is rather crude and usually underestimates the effect. Elongation acts as to reduce electromagnetic effects which, in turn, tend to increase the toroidal momentum pinch.

Finally, we note that the height of the density pedestal and the core density are considerably smaller than those in the experiment, while the temperatures are considerably larger. Actually, there is a particle pinch in the system which works on a very long timescale as seen in Ref. 4. It will eventually lead to a higher density which then also leads to lower temperatures. Of course there is some physics missing close to the scrape off layer. There was no data for increased impurity content near the separatrix and atomic physics has not been included. The height of the density barrier also depends somewhat on the gas puffing rate. We note that these simulations are quite complicated. The L-H transition in JET (Ref. 24) was simulated with fixed density kept at the pedestal level and also there an L-H transition of the temperatures was obtained.
From these points of view, we can, to some extent, regard these simulations as general simulations of the plasma physics processes included in the $\mathrm{L}-\mathrm{H}$ transition where also global effects are included. We have addressed several, so far not understood, general aspects of the L-H transition such as the dependence of the power threshold on the direction of the grad B drift, the increase of the power threshold with magnetic field, and the fact that the density gets much flatter in $\mathrm{H}$-mode. Also the agreement with the turbulence simulations by Rogers et al., are not related to a particular machine as the work by Rogers $e t$ al. is not in itself although it shows good agreement with $\mathrm{C}$-mod. Agreement with a turbulence code also further strengthens our confidence in the nonlinear saturation mechanisms included in the model as discussed after Eq. (3). An additional point here is the agreement with the linear scaling of $\chi_{\mathrm{i}}$ with the distance to the threshold in $\eta_{\mathrm{i}}$ (Ref. 24) as found also by Hamaguchi and Horton in turbulence simulations. ${ }^{43}$ The fluid aspects of the model are further supported by a coming paper in Journal of Plasma Physics. ${ }^{44}$ We note also that Zonal flows are strongly nonlinear effects due to wave intensities so strongly nonlinear effects are actually included here. Concerning Edge Localized Mode (ELMs) there has in some cases been evidence for nonstationarity in well developed $\mathrm{H}$ modes. Since both kinetic ballooning modes and peeling modes are included, we expect that the driving force of ELMs is included. Averaging over ELMs could, of course, very well modify our temperature and density levels.

The author is grateful to Chuan S. Liu for pointing out unsolved questions and for continuous encouragement. The author is grateful to the EAST team for providing the original data and to Arnold Kritz and the Lehigh group for providing TRANSP data for EAST38300. Thanks are also due to both the SWIP and ASIPP teams for comments during presentations made in Chengdu and Hefei. Thanks are also due to Hans-George Gustavsson for find assistance with the Fortran plotpackage.

The author acknowledges support from CAS and ASIPP for visits to ASIPP during which parts of this work was done.

${ }^{1}$ B. Wan, EAST and HT-7 Teams, and International Collaborators, "Recent experiments in the EAST and HT-7 superconducting tokamaks," Nucl. Fusion 49, 104011 (2009))

${ }^{2}$ J. Li, B. Wan, and the EAST Team and International Collaborators, "Recent progress in RF-heating and long pulse experiments on EAST," Nucl. Fusion 51, 094007 (2011).

${ }^{3}$ G. S. Xu, B. N. Wan, J. G. Li, X. Z. Gong, J. S. Hu, J. F. Shan, H. Li, D. K. Mansfield, D. A. Hupreys, V. Naulin, and EAST Team and International Collaborators, "Study on H-mode access at low density with lower hybrid current drive and lithium wall-coatings on the EAST superconducting tokamak," Nucl. Fusion 51, 072001 (2011).

${ }^{4}$ G. Q. Wang, J. Ma, J. Weiland, and Q. Zang, Phys. Plasmas 20, 102310 (2013).

${ }^{5}$ F. Wagner, G. Becker, K. Behringer, D. Campbell, A. Eberhagen, W. Engelhardt, G. Fussmann, O. Gehre, J. Gernhardt, G. V. Gierke et al., Phys. Rev. Lett. 49, 1408 (1982).

${ }^{6}$ K. H. Burrell, S. Allen, G. Bramsen, N. H. Brooks, R. W. Callis, T. N. Carlstrom, M. S. Chu, A. P. Colleraine, D. Content, J. C. DeBoo et al., Plasma Phys. Controlled Fusion 31, 1649 (1989). 
${ }^{7}$ K. H. Burrell, T. N. Carlstrom, E. Doyle, P. Gohil, R. J. Groebner, T. Lehecka, T. Luhman, H. Matsumoto, T. H. Osborne, W. A. Peebles, and R. Philipona, Phys. Fluids B 2, 1405 (1990).

${ }^{8}$ JET Team, Presented by D. Stork, in Proceeding of 14th International Conference on Plasma Physics and Controlled Nuclear Fusion Research, 1992, Wurtzburg (IAEA, Viennam, 1993), Vol. 1, p. 725.

${ }^{9}$ J. E. Rice, E. S. Marmar, F. Bombarda, and L. Qu, Nucl. Fusion 37, 421 (1997).

${ }^{10}$ A. E. Hubbard, R. L. Boivin, J. F. Drake, M. Greenwald, Y. In, J. H. Irby, B. N. Rogers, and J. A. Snipes, Plasma Phys. Controlled Fusion 40, 689 (1998).

${ }^{11}$ A. E. Hubbard, J. W. Hughes, I. O. Bespamyatnov, T. Biewer, I. Cziegler, B. LaBombard, Y. Lin, R. McDermott, J. E. Rice, W. L. Rowan, J. A. Snipes, J. L. Terry, S. M. Wolfe, S. Wukitch, and the Alcator C-Mod Group, Phys. Plasmas 14, 056109 (2007).

${ }^{12}$ P. C. deVries, K. M. Rantamäki, C. Giroud, E. Asp, G. Corrigan, A. Eriksson, M. deGreef, I. Jenkins, H. C. M. Knoops, P. Mantica, H. Nordman, P. Strand, T. Tala, J. Weiland, K.-D. Zastrow, and the JETEFDA Contributors, Plasma Phys. Controlled Fusion 48, 1693 (2006).

${ }^{13}$ ITER Physics Basis Editors et al., "Progress in ITER Physics basis," Nucl. Fusion 47, S1-S413 (2007).

${ }^{14}$ J. W. Connor, R. J. Hastie, H. R. Wilson, and R. L. Miller, Phys. Plasmas 5, 2687 (1998).

${ }^{15}$ J. W. Connor and H. R. Wilson, Plasma Phys. Controlled Fusion 42, R1-R74 (2000).

${ }^{16}$ P. N. Guzdar, J. F. Drake, D. McCarty, A. B. Hassam, and C. S. Liu, Phys. Fluids B 5, 3712 (1993).

${ }^{17}$ B. N. Rogers, J. F. Drake, and A. Zeiler, Phys. Rev. Lett. 81, 4396 (1998).

${ }^{18}$ R. Singh and J. Weiland, Phys. Plasmas 6, 1397 (1999).

${ }^{19}$ R. Moestam and J. Weiland, Nucl. Fusion 42, 663 (2002).

${ }^{20}$ M. N. A. Beurskens, A. Alfier, B. Alper, I. Balboa, J. Flanagan, W. Fundamenski, E. Giovanossi, M. Kampenaars, A. Loarte, P. Lomas et al., Nucl. Fusion 49, 125006 (2009).

${ }^{21}$ P. B. Snyder, R. J. Groebner, A. W. Leonard, T. H. Osborne, and H. R. Wilson, Phys. Plasmas 16, 056118 (2009)

${ }^{22}$ C. Z. Cheng, Phys. Fluids 25, 1020 (1982).

${ }^{23}$ P. Andersson and J. Weiland, Phys. Fluids 31, 359 (1988).

${ }^{24}$ J. Weiland, Stability and Transport in Magnetic Confinement Systems (Springer, New York, 2012).
${ }^{25}$ T. S. Hahm, P. H. Diamond, O. D. Gurcan, and G. Rewoldt, Phys. Plasmas 14, 072302-1 (2007).

${ }^{26}$ D. Strinzi, A. G. Peeters, and J. Weiland, Phys. Plasmas 15, 044502 (2008).

${ }^{27}$ J. Weiland, R. Singh, H. Nordman, P. K. Kaw, A. Peeters, and D. Strintzi, Nucl. Fusion 49, 065033 (2009).

${ }^{28}$ R. E. Waltz, G. O. Kerbel, and J. Milovich, Phys. Plasmas 1, 2229 (1994).

${ }^{29}$ A. Zagorodny and J. Weiland, Phys. Plasmas 16, 052308 (2009).

${ }^{30}$ G. M. Staebler, R. E. Waltz, and J. C. Wiley, Nucl. Fusion 37, 287 (1997).

${ }^{31}$ R. E. Waltz, R. R. Dominguez, and G. W. Hammett, Phys. Fluids B 4, 3138 (1992).

${ }^{32}$ A. Mahmood, A. Eriksson, and J. Weiland, "Electromagnetic effects on toroidal momentum transport," Phys. Plasmas 17, 122310 (2010).

${ }^{33}$ J. Weiland, Phys. Plasmas 11, 3238 (2004).

${ }^{34}$ J. Weiland and I. Holod, Phys. Plasmas 12, 012505 (2005).

${ }^{35} \mathrm{~J}$. Weiland, P. Mantica, and the JET-EFDA Contributors, in 38th EPS Conference, Strasbourg, France, June 27-July 1 2011, Paper No. P5.130.

${ }^{36}$ J. Weiland, K. Crombe, P. Mantica, V. Naulin, T. Tala, and the JETEFDA Contributors, "IFP-CNR - Chalmers Workshop on nonlinear phenomena in fusion plasmas," AIP Conf. Proc. 1392, 85 (2011).

${ }^{37}$ S. Novakovskii, P. Guzdar, J. Drake, C. S. Liu, and F. Waelbroeck, Phys. Plasmas 2, 3764 (1995).

${ }^{38}$ K. Crombe, Y. Andrew, M. Brix, C. Giroud, S. Hacquin, N. C. Hawkes, A. Murari, M. F. F. Nave, J. Ongena, V. Parail, G. Van Oost, I. Voitsekhovich, and K.-D. Zastrov, Phys. Rev. Lett. 95, 155003 (2005).

${ }^{39}$ J. E. Rice, A. Ince-Cushman, J. S. DeGrassie, L. G. Eriksson, Y. Sakamoto, A. Scarabosio, A. Botholon, K. H. Burrell, B. P. Duval, C. Fenzi-Bonizek et al., Nucl. Fusion 47, 1618 (2007).

${ }^{40}$ A. Peeters, C. Angioni, A. Bortholon, Y. Camenen, F. J. Casson, B. Duval, L. Fiederspiel, W. A. Hornsby, Y. Idomura, T. Hein et al., Nucl. Fusion 51, 094027 (2011).

${ }^{41}$ F. Jenko, W. Dorland, and G. W. Hammett, Phys. Plasmas 8, 4096 (2001).

${ }^{42}$ T. Rafiq, A. Kritz, J. Weiland, A. Y. Pankin, and L. Luo, Phys. Plasmas 20, 032506 (2013).

${ }^{43}$ S. Hamaguchi and W. Horton, Phys. Fluids B 2, 1833 (1990).

${ }^{44}$ J. Weiland, C. S. Liu, and A. Zagorodny, "Transition from a coherent three-wave system to turbulence with application to the fluid closure," J. Plasma Phys. Published online June 09 (2014). 